\title{
Effect of oxidation degrees of graphene oxide (GO) on the structure and physical properties of chitosan/GO composite films
}

\begin{abstract}
Graphene oxide (GO) samples with different oxidation degrees were prepared using the modified and improved Hummers' method by varying the ratios of graphite to potassium permanganate $\left(\mathrm{KMnO}_{4}\right)$ at 1:2 (GO1), 1:4 (GO2), 1:6 (GO3), and 1:8 (GO4). Fourier-transform infrared spectroscopy (FTIR) and Raman spectroscopy confirmed the increase in oxygencontaining groups in $\mathrm{GO}$ with the increase in $\mathrm{KMnO}_{4}$ concentration. Composite films comprising chitosan (CS) and GO were synthesized using the solution casting method and were characterized by mechanical tests, scanning electron microscopy (SEM), FTIR spectroscopy, and contact angle analysis. GO4 yielded a significant $(\mathrm{p}<0.05)$ improvement in mechanical strength and lower UV light transmission without significantly $(p \geq 0.05)$ increasing the water vapor permeability (WVP), compared to a neat CS film. Films with GO4 also had denser structure and all films were completely degradable within 20 days in soil compost. This study presents the effect of GO oxidation degrees on the properties of CS/GO composite films as potential materials for food packaging.
\end{abstract}

Keyword: Graphene oxide; Oxidation; Chitosan; Nanocomposite films; Biodegradability 